\title{
Restricción alimentaria y sobrealimentación: Un modelo de la neurociencia afectiva
}

\author{
Jaime R Silvaa. \\ O vereating and restrained eaters. \\ An affective neuroscience perspective
}

Some subjects may overeat when they experience anxiety, even if they chronically restrict their food consumption. This contradictory behavior is a dysfunctional emotional regulation mechanism, which promotes the development of obesity and eating disorders. We review studies from a research program where alimentary restriction and overeating are conceived under the perspective of affective neuroscience. In this framework, restrained eaters $(\mathrm{RE})$ are described as subjects that are emotionally vulnerable and have dysfunctional emotional modulation strategies. We discuss empiric evidence about the influence of motivational systems on alimentary behavior. Electrophysiological observations in RE reveal a self-referential processing of food stimulus as well as dysfunctional processing during the differentiation of emotional expressions. We stress the role of emotional education and the creation of psychometric instruments designed for early detection of restrained eaters (Rev Méd Chile 2008; 136: 1336-42).

(Key words: Anxiety; Eating disorders; Neurosciences; Restrained eaters)

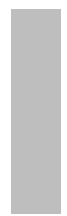

Recibido el 26 de julio, 2007. Aceptado el 26 de noviembre, 2007. Actualizado el 26 de agosto, 2008.

Financiamiento: Dirección de Investigación de la Universidad de La Frontera (DIUFRO).

Departamento de Salud Mental y Psiquiatría, Facultad de Medicina, Universidad de La Frontera, Temuco, Chile.

a Psicólogo, PhD. en Psicobiología

\section{EMOCIONES Y SOBREALIMENTACIÓN:}

EL CASO DE LOS DIETANTES CRÓNICOS

L a relación entre el patrón alimenticio de un individuo y el estrés ha sido una prolífica área de investigación en las ciencias de la conducta y la medicina ${ }^{1,2}$. Aunque se ha comunicado que el

Correspondencia a: Dr. Jaime R. Silva. Departamento de Salud Mental y Psiquiatría, Facultad de Medicina, Universidad de La Frontera. Manuel Montt 112, Temuco. Región de la Araucanía. Fono-Fax: (45) 325 736. E mail: jaimesilva@ufro.cl estrés induce un decremento de la alimentación ${ }^{3}$, varios estudios sugieren que los niveles de ingesta dependen de una interacción entre las características psico-afectivas de los sujetos y el tipo de estrés involucrado ${ }^{4-7}$. Lo anterior ha resultado especialmente importante para las ciencias de la salud, ya que la relación entre restricción, sobrealimentación y estrés tiene importantes implicancias para los desórdenes de la alimentación (i.e. anorexia nervosa y bulimia nervosa) ${ }^{2}$ y la obesi$\operatorname{dad}^{8,9}$. Dichas alteraciones representan el objeto de estudio de innumerables grupos de investigación en el mundo ${ }^{2}$ y Chile, como los de Be- 
har $^{10,11}$, Cordella ${ }^{12}$, Kain ${ }^{13}$, Lolas ${ }^{14}$, y de investigación teórica como los de Peña y Lillo ${ }^{15}$ y Figueroa ${ }^{11}$, por nombrar algunos.

En este contexto, un grupo específico de individuos, que muestran una particular combinación de restricción alimentaria y sobrealimentación, han sido profundamente estudiados por diversos equipos de investigación. Por sus hábitos alimenticios, han sido denominados comedores refrenados (restrained eaters) ${ }^{4,8}$ o dietantes crónicos (DC) ${ }^{16}$ y pueden ser caracterizados de modo general como personas que presentan un temor exacerbado a ganar peso, restringiendo su alimentación por medio de dietas ${ }^{4}$. Paradójicamente, bajo ciertas condiciones, estos individuos aumentan sus niveles de ingesta, sobrealimentándose $e^{1,2,4-9}$. Tales condiciones generalmente incluyen el consumo de una comida "prohibida", la ingesta de alcohol o estados emocionales intensos en respuesta a estresores ambientales ${ }^{6}$.

Los estados de ánimo y las emociones parecen jugar un rol importante en el consumo de alimentos en personas normales. Por ejemplo, diversos estudios muestran que comúnmente un individuo disminuye significativamente el monto de alimento cuando experimenta emociones negativas ${ }^{17-19}$. Sin embargo, para los DC algunas condiciones emocionales ejercen una influencia opuesta, favoreciendo el incremento del nivel de consumo de alimento. De hecho, se ha reportando desinhibición alimentaria en DC bajo múltiples estados afectivos. El debate, en este contexto, se ha centrado en develar; a) cuál dimensión de la emoción (i.e. valencia vs intensidad) es el aspecto más influyente como fuente de desinhibición y; b) qué emociones en particular explican las alteraciones de la alimentación observadas en los DC.

Se ha propuesto que el nivel de activación afectivo o arousal, facilita la desinhibición alimentaria del DC independientemente de la valencia emocional (positiva o negativa). Es decir, bastaría que un DC experimentara un estado emocional lo suficientemente intenso para que se expresara su tendencia a sobrealimentarse. Sin embargo, existe evidencia que ciertas condiciones específicas deben ocurrir para que los estados emocionales desencadenen la desinhibición alimentaria en $\mathrm{DC}^{19-21}$, condiciones asociadas principalmente a emociones negativas y no a las positivas.

En los DC el quiebre de la dieta es especialmente pronunciado en condiciones de ansiedad donde una fuente anticipada de estrés amenaza a la identidad personal y la autoestima1,7,20,21. De esta manera, la combinación de la ansiedad y la amenaza de la identidad es el ingrediente fundamental de la sobreingesta en los DC. Consecuentemente, el rol del self se ha destacado en el debate teórico en torno a los patrones alimenticios anormales de los DC. Se han ofrecido diversas hipótesis respecto de este proceso, donde aquellas que involucran a la identidad han recibido mayor respaldo empírico ${ }^{1}$.

\section{HIPÓTESIS EXPLICATIVAS \\ DE LA CONDUCTA ALIMENTARIA DE LOS DC}

Han sido propuestos varios modelos explicativos de la conducta alimentaria del DC, especialmente de la combinación particular de restricción/sobrealimentación. Entre las explicaciones que mayor atención científica e investigación han generado se encuentra la hipótesis de confort psicosomático ${ }^{22,23}$, la hipótesis de la distractibili$\operatorname{dad}^{24}$, la hipótesis de enmascaramiento ${ }^{1,25}$ y los modelos asociados al self en general ${ }^{1,7,26}$. Estos últimos han recibido mayor apoyo empírico ${ }^{1}$.

La hipótesis del confort psicosomático plantea que los dietantes calman o disminuyen la experiencia de ansiedad mediante la comida. Por ello, en condiciones de ansiedad estos sujetos tenderían a aumentar sus niveles de ingesta. Según esta aproximación la comida cumplińa una función "ansiolítica" que permitina a los DC controlar estos sentimientos.

La hipótesis de distracción sugiere que los DC utilizanían el alimento para distraerse de las emociones negativas. El alimento y su consumo permitinan al individuo focalizar su atención fuera de los contenidos mentales amenazantes asociados a la ansiedad, al menos durante el periodo de tiempo que implica el consumo. Así, a mayor necesidad de distraerse de la experiencia de ansiedad, durante mayor tiempo se requerirá consumir alimento, aumentando la ingesta y generando la sobrealimentación. Los modelos centrados en el self o la identidad han propuesto que la sobrealimentación es una forma de regulación de los afectos asociados a una autoimagen negativa, mediante un cambio en el foco de atención (esto último con matices según cada aproximación). Destacan en este ámbito las propuestas de Heatherton y Baumeister $^{7}$, Polivy y Herman ${ }^{1}$ y Wallis y Hetherington ${ }^{26}$. 
Heatherton y Baumeister ${ }^{7}$ sostienen que los DC generalmente tienen una mala imagen de sí mismos (especialmente de su apariencia física), por lo cual intentanian evitar estos sentimientos displacenteros constriñendo su atención a la situación inmediata (alimento). Este escape de autoconciencia negativa ayuda a los dietantes a "olvidar" momentáneamente su imagen negativa, alejándose de un planeamiento de largo plazo de su conducta y debilitando la inhibición que generalmente los restringe ${ }^{27}$. Polivy y Herman ${ }^{1}$ enfatizan el hecho que los DC atribuyen emóneamente la ansiedad relacionada con la amenaza de la autoestima, reinterpretándola como angustia por haber roto la dieta. La ansiedad estanía aún presente pero interpretada de una manera preferible; de una situación vivida como sin control a una situación potencialmente controlable ("mañana empiezo la dieta"). Por esta razón, estos autores afirman que el quiebre de restricción alimentaria es una forma de "enmascarar" la ansiedad y la amenaza al self. En un estudio reciente, Wallis y Hetherington ${ }^{26}$ sugieren que la amenaza de la identidad dificultańa el monitoreo de la restricción alimentaria debido a que los recursos cognitivos deben focalizarse en una tarea demandante de atención. Así, el debilitamiento del control ejecutivo dificultańa la regulación alimentaria de largo plazo.

Los estudios del self en DC muestran que estos sujetos requieren modular sus estados afectivos negativos asociados a la amenaza de la autoimagen, enmascarando/distrayendo el origen de la ansiedad mediante la atribución de esos estados negativos al rompimiento de la dieta. Sin embargo, este mecanismo no tendría como resultado la eliminación de la experiencia emocional negativa sino que probablemente modifica la sensación de control sobre el evento adverso, de ser una situación incontrolable (el fracaso) a una controlable (el hacer dieta) ${ }^{7}$. Una revisión en detalle de estas teorías y los estudios empíricos relacionados puede obtenerse en el reporte de Silva ${ }^{28}$.

\section{PsiCOFISIOLOGÍA Y RESTRICCIÓN ALIMENTARIA: UN MODELO DE LA NEUROCIENCIA AFECTIVA}

Como se mencionó anteriormente, las características conductuales de los DC han sido estudiadas por aproximaciones denivadas de teonías psicológicas de la atención, la emoción y la modulación de la identidad. Si bien existe un cuerpo de conocimiento bastante amplio respecto de las condiciones que favorecen la sobrealimentación y las caracteństicas psicológicas de los DC, sólo recientemente se ha utilizado la psicofisiología como marco conceptual y metodológico ${ }^{\mathrm{a}}$. El equipo de investigación que he liderado ha descrito, a partir de un modelo teónico de la neurociencia afectiva, ciertos marcadores psicofisiológicos asociados a las disposiciones afectivas de los DC. Más aún, se ha elaborado un modelo para la comprensión de la influencia del estilo afectivo en la conformación de este patrón alimenticio alterado. A continuación se describirá esta aproximación.

El estilo afectivo corresponde al rango de diferencias individuales en los múltiples componentes de las disposiciones anímicas y la reactividad afecti$\mathrm{va}^{29}$. Las estructuras cerebrales que subyacen al estilo afectivo conforman dos sistemas que sustentan la motivación y emoción: el sistema de aproximación conductual y el sistema de inhibición 0 evitación conductual ${ }^{30}$. El sistema de aproximación favorece las conductas apetitivas y genera afectos positivos relacionados al logro de metas (entre otros). El sistema de evitación facilita al organismo el distanciamiento de una fuente aversiva de estimulación y organiza las respuestas apropiadas cuando es confrontado con estímulos amenazantes. Los componentes fundamentales del sistema de aproximación son la corteza prefrontal, los ganglios de la base y el núcleo accumbens. El sistema de evitación, a su vez, estanía compuesto fundamentalmente por la corteza prefrontal y la amígdala ${ }^{30}$. En concordancia con los estudios de la estructura del afecto ${ }^{16}$, las investigaciones de la neurociencia afectiva sugieren que los sistemas motivacionales son ortogonales, mostrando una segregación parcial a nivel cortical. Específicamente, la corteza prefrontal derecha estanía directamente implicada en el sistema de evitación, mientras que la izquierda lo estanía del sistema de aproximación ${ }^{29}$. Congruente con esta formulación, las diferencias en la actividad tónica de estos componentes, así como su relación funcional, representan el sustrato biológico del estilo afectivo ${ }^{16,29,30}$.

Bajo el alero de este marco conceptual, hemos indagado la participación de los sistemas motiva-

aExisten algunas excepciones como el programa de investigación chileno del Dr. Lolas; FONDECYT 1961083. 
cionales en algunos aspectos de los DC. En un primer estudio realizado en el Laboratory for Affective Neuroscience ${ }^{31}$, demostramos que los DC pueden caracterizarse como sujetos con una asimetría funcional derecha de la corteza prefrontal. Tal como hemos sostenido, ello implica que las personas que realizan dieta crónicamente poseen un sistema de motivación de inhibición más activo y, por ende, preponderante en la organización de la conducta dirigida a metas. Ello implicaría que los comedores refrenados poseen un estilo afectivo congruente con dicho marcador psicofisiológico, principalmente una vulnerabilidad al estrés y una dificultad en regular las emociones negativas $^{16,29}$. En este sentido, sostenemos que en el DC se daría una combinación entre una disposición emocional determinada (tendencia exacerbada a experimentar ansiedad en condiciones de novedad o estrés), en conjunto con un mecanismo de regulación específico (la sobrealimentación). Sin embargo, esta observación debe ser complementada con datos provenientes de un segundo estudio $^{32}$, el cual deriva de conceptualizaciones previas directamente relacionadas con la asimetría de los sistemas motivacionales ${ }^{8,16}$. Debido al reporte relativo a que algunos dietantes crónicos muestran un patrón de asimetría de los sistemas motivacionales diferente (preponderancia del sistema de aproximación), probamos la hipótesis de una influencia ponderada de estos sistemas en la tendencia a sobrealimentarse 0 restringirse exhibida por los DC. En condiciones que favorecen la sobrealimentación, mostramos que los DC con un sistema de evitación más activo se sobrealimentan pero que aquellos con un sistema de aproximación sensibilizado tienden a restringirse significativamente en su alimentación. Por ello, tal como he sostenido 8,32 , esta conceptualización permite especular que, en la medida que la condición de restricción crónica de la alimentación se ha asociado a la obesidad y algunos trastornos alimentarios, como la anorexia nervosa, es probable que la combinación específica de un determinado estilo afectivo (asimetría de los sistemas motivacionales) con la regulación de los afectos a través de la alimentación, delimite sendas específicas del desarrollo evolutivo de dichos trastornos. En otras palabras, dado que los DC con un sistema de aproximación sensible tienden a exacerbar su restricción durante condiciones que favorecen la desinhibición, es posible predecir que dichos sujetos en condiciones crónicas de estrés tendrán patrones alimenticios crónicamente restringidos, favoreciendo la aparición de desórdenes alimentarios como la anorexia. Para el DC con un sistema de evitación sensibilizado se esperaría el patrón opuesto, es decir frente a el estrés crónico estos sujetos, al responder mediante la sobrealimentación para regular sus afectos, se generarían las condiciones para el desarrollo de obesidad.

Dos estudios adicionales ${ }^{33,34}$ nos han permitido delinear con mayor exactitud algunos elementos del estilo afectivo de los DC. En primer lugar, en condiciones de ansiedad, los DC mostraron un procesamiento cortical de los estímulos alimenticios anormal. Tal como predice el modelo de enmascaramiento, a diferencia de los controles, en individuos DC los estímulos alimenticios reclutan estructuras corticales de la línea media, las cuales han sido recientemente implicadas en el procesamiento autorreferencial de estímulos y la autoimagen ${ }^{35,36}$. Más aún, este procesamiento se acompañó con una sensibilización de las redes occipitales relacionadas con la atención motivada. Silva y Pizzagalli sugieren que el proceso autorreferencial activado en respuesta a la comida sería un aspecto esencial del procesamiento de información de los DC en condiciones de ansiedad ${ }^{33}$. Finalmente, en otro estudio de potenciales cerebrales ${ }^{34}$, nuestro equipo de investigación develó alteraciones en el procesamiento de expresiones emocionales en DC. En acuerdo con la creciente evidencia que sostiene que la diferenciación emocional es un aspecto esencial en la regulación afectiva, los DC muestran patrones corticales occipitales indiferenciados frente a rostros expresivos emocionales. De hecho los controles muestran una progresión de la activación cortical significativamente distinta para expresiones neutras, positivas y negativas. Esta evidencia ha sido interpretada como un marcador psicofisiológico de las dificultades que los DC para diferenciar emociones, lo cual favorecenía el desarrollo de mecanismos disfuncionales de modulación afectiva (sobrealimentación/restricción).

En síntesis, según la propuesta presentada, los DC serían personas con un sistema motivacional sensibilizado que se expresa en alteraciones del estilo afectivo (particularmente en la sensibilidad frente al estrés y dificultades en la diferenciación emocional), incluyendo el desarrollo de mecanis- 
mos de regulación emocional disfuncionales (asociado a un procesamiento autorreferencial de estímulos alimenticios). Tal como se representa didácticamente en la Figura 1 los DC poseerían un determinado estilo afectivo que representaría una diátesis para los trastornos de la alimentación y la obesidad, el cual se expresaría en condiciones de estrés (agudo o crónico). El nivel de diferenciación emocional (representado por el tamaño del cono), determinaría las capacidades del individuo para elaborar estrategias de regulación afectiva, donde a menor nivel de diferenciación mayor probabilidad de elaboración de estrategias disfuncionales (entre ellas la sobrealimentación/restricción).

Aunque podría considerarse prematuro y debiendo aún documentar la eficiencia/eficacia de los programas preventivos, en la siguiente sección se abordan los posibles aportes del programa de trabajo presentado para futuras políticas de salud, en el entendido que se trata de una aproximación provisoria, que forma parte de un proyecto en curso de largo plazob.
DisCUSIÓN: APORTES DEL PROGRAMA DE INVESTIGACIÓN PARA FUTURAS POLÍTICAS DE SALUD Y EL DESARROLLO DE ACCIONES PREVENTIVAS

Tal como se presentó anteriormente, la aproximación de la neurociencia afectiva para la comprensión de los DC se encuentra en una fase de desarrollo, principalmente a través de estudios experimentales de laboratorio. Sin embargo, en la medida que esta propuesta alcanza un grado de madurez suficiente y que los datos continúan armonizando con los modelos planteados, se hace necesario transitar de la ciencia básica a la ciencia aplicada.

En este contexto se debe considerar que la relevancia del estudio de los $\mathrm{DC}$, tal como se ha mencionado, es su rol en el desarrollo de trastornos de la alimentación y la obesidad. Así como otras enfermedades de la salud mental (i.e. trastorno depresivo mayor), los trastornos de la alimentación y la obesidad (esta última una condición médica), probablemente son la expresión superficial común de mecanismos etiológicos diferentes.
Figura 1. Estilo afectivo y sobrealimentación. Un modelo de la neurociencia afectiva.

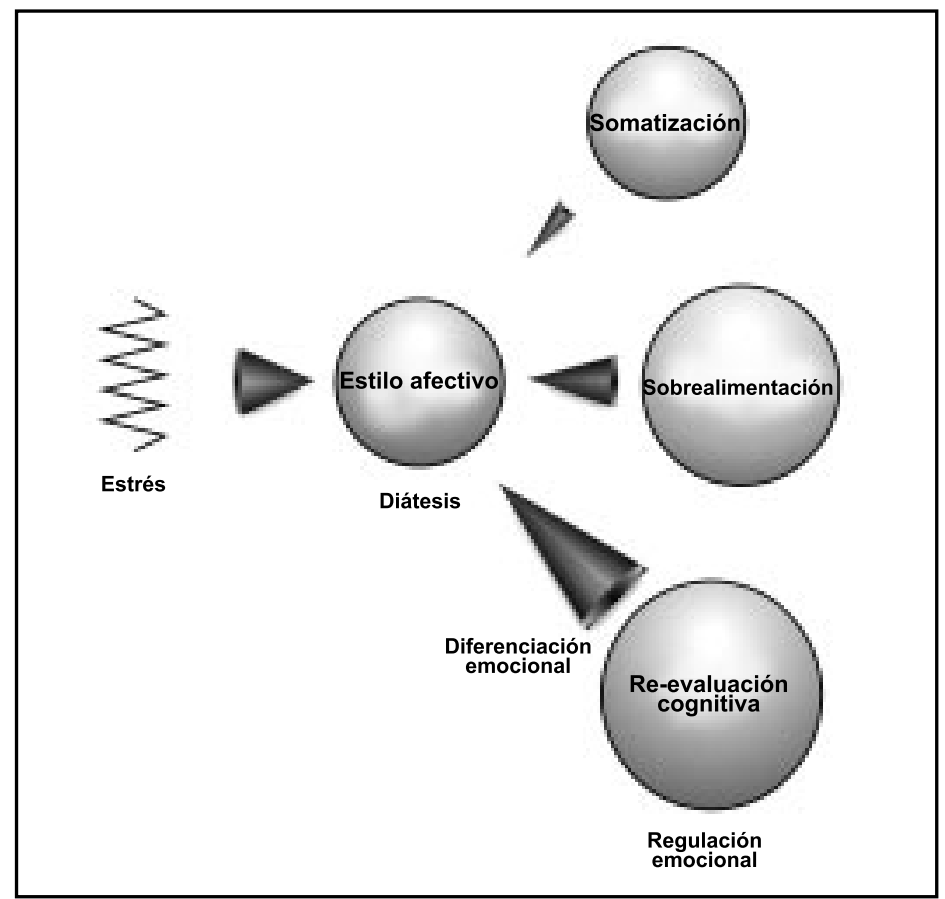

$\overline{\mathrm{b}} \mathrm{El}$ autor agradece a un revisor anónimo las sugerencias que dieron origen a este párrafo. 
En este marco, el estudio de los DC permite; a) conocer los mecanismos etiológicos de una vía específica para el desarrollo de alguno de esos trastornos y, en consecuencia; b) la formulación de estrategias de prevención e intervención para una población particular de individuos.

Así, son varios los niveles de análisis posibles de establecer a partir de esta propuesta. Uno de los aspectos más relevantes, desde el punto de vista de la salud mental, es el desarrollo de acciones preventivas en la población. Considerando los mecanismos que originan el patrón alterado de alimentación, que afectan incluso el control cognitivo del comportamiento ${ }^{37}$, es posible sostener que el establecimiento de políticas que favorezcan el desarrollo de capacidades de modulación afectiva en la población generaría un factor protector de importancia para este tipo de alteraciones. Recordemos que el DC es un sujeto que tiene una vulnerabilidad al estrés, en la cual se ha desarrollado un mecanismo de regulación emocional disfuncional. En el transcurso del ciclo vital es probable que los estresores que el individuo enfrenta estén circunscritos a eventos discretos. Sin embargo, cuando el estrés adquiere un carácter crónico, los mecanismos de modulación basados en la restricción/sobrealimentación pueden generar importantes alteraciones de la alimentación del individuo ${ }^{34}$. El aumento de las capacidades de diferenciación emocional tiene un efecto sobre la regulación de los afectos, donde el aumento de la capacidad de distinción emocional (o "granulidad afectiva"), aumenta la efectividad de la regulación de las emociones negativas ${ }^{16}$. En los comedores refrenados sería deseable inducir una transición desde una regulación de los afectos basados en mecanismos disfuncionales hacia otros más complejos y funcionales (como la reevaluación cognitiva ${ }^{38}$ ). Así, la educación emocional es un aspecto central a considerar respecto de las alteraciones de la conducta alimentaria de los DC. Esta lógica es aplicable también a la elaboración de estrategias de intervención en pacientes DC que presentan alteraciones significativas de la alimentación. Para una revisión en extenso de este punto y la descripción de principios fundacionales para la psicoterapia y prevención, ver el trabajo de Silva $^{34}$ y Silva y Slachevsky ${ }^{38}$.
Por otro lado, la detección temprana de los sujetos DC es una promisoria forma de abordar los desórdenes de la alimentación y la obesidad. Debido a que el DC es una vía de desarrollo para las alteraciones descritas, uno de los campos de aplicación del modelo presentado es la detección de los individuos que tienen la combinación de vulnerabilidad emocional y el mecanismo disfuncional de regulación emocional de restricción/sobrealimentación. Lamentablemente, son escasos los instrumentos psicométricos que abordan confiablemente esta temática en sujetos menores de 15 años, sin embargo, la experiencia del grupo de investigación de Kain ${ }^{39}$ es instructiva al respecto. Así, una de las primeras tareas en esta área es la creación de cuestionarios válidos y confiables que permitan económicamente distinguir la población diana, principalmente en la infancia y la niñez. A partir de ello, también será posible abordar experimentalmente qué tipo de vínculos afectivos tempranos se relacionan con la generación de patrones de restricción/ sobrealimentación característico de los DC y qué aspectos de la educación/interacción familiar favorece el desamollo de los mecanismos disfuncionales de regulación de los afectos en el DC.

El estudio de los patrones de vulnerabilidad/ resiliencia de los $\mathrm{DC}^{40} \mathrm{y}$ la detección temprana y las acciones preventivas aquí descritas, permitirá elaborar políticas de salud pública acordes con las características de los sujetos DC y, de este modo, abordar una de las sendas de desarrollo de trastornos de la alimentación y obesidad. Un desafío de esta naturaleza exige la acción colaborativa de los investigadores y profesionales de la salud mental en su más amplio sentido.

\section{Agradecimientos}

A los miembros de la Unidad de Trastornos Alimenticios y Obesidad, Clínica Alemana de Temuco y a su Directora Dra. Marcela Jiménez, por motivar la elaboración de este artículo. A los Drs. Richard J. Davidson, Diego A. Pizzagalli, Chris Larson y Andrea Slachevsky C. por su invaluable colaboración en parte de los estudios mencionados en este artículo. A los psicólogos Jorge del Canto, Noemi Sanagua, Amine Latif, Raúl Jiménez, Gabriel Reyes y Emesto Guerra, quienes han colaborado en los últimos 4 años en el trabajo de laboratorio. Finalmente a la Dra. Patricia Cordella por sus valiosos comentarios, sugerencias y por la minuciosa revisión de la versión inicial del texto. 


\section{REFERENCIAS}

1. Polvy J, Herman P. Distress and eating: why do dieters overeat? Int J Eat Disord 1999; 26: 153-64.

2. Polvy J, Herman P. Causes of eating disorders. Annu Rev Psychol 2002; 53: 187-213.

3. Schachter S, Goldman R, Gordon A. Effects of fear, food deprivation, and obesity on eating. J Pers Soc Psychol 1968; 10: 91-7.

4. Herman P, Polivy J. Restrained eating. En: Stunkard A, ed. Obesity. Philadelphia: Saunders 1980; 208-25.

5. Polivy J, Herman P. Dieting and binding: a causal analysis. Am Psychol 1985; 40: 193-201.

6. RUDERMAN A. Dietary restraint: a theoretical and empirical review. Psychol Bull 1986; 99: 247-62.

7. Heatherton T, Baumeister R. Binge-eating as escape from self-awareness. Psychol Bull 1991; 126: 890-900.

8. SiLva J. Ansiedad y sobrealimentación. Investig Cienc 2005; 341: 33-4.

9. JевB S. Aetiology of obesity. Br Med Bull 1997; 53: 264-85.

10. Behar R, Manzo R, Casanova D. Trastornos de la conducta alimentaria y asertividad. Rev Méd Chile 2006; 134, 312-19.

11. Behar R, Figueroa G, ed. Anorexia nerviosa y bulimia. Clínica y terapéutica. Santiago de Chile: Mediterráneo, 2004.

12. Cordelia $P$, Lizana $P$, Urrejola $P$, Figueroa D, Del Río $P$, Hodgson MI ET AL. Programa de transtornos de alimentación en adolescentes y jóvenes chilenos: variaciones a seis meses de tratamiento. Rev Méd Chile 2006; 134: 973-80.

13. Kain J, LeRa L, Rojas J, UauY R. Obesidad en preescolares de la Región Metropolitana de Chile. Rev Méd Chile 2007; 135, 63-70.

14. PeÑa y LuLo S. La manía de adelgazar. Santiago de Chile: Santiago, 1997.

15. Lolas F, SAnfuentes MT, Liberman C. Feeding behavior, obesity and physiologic mechanisms. Acta Psiquiatr Psicol Am Lat 1989; 35, 94.

16. SiLva J. Biología de la regulación emocional: su impacto en la psicología del afecto y la psicoterapia. Ter Psicol 2003; 21: 163-72.

17. Schotte DE, Cools J, Mcnaliy RJ. Film-induced negative affect triggers overeating in restrained eaters. J Abnorm Psychol 1990; 99: 317-20.

18. BAUCON D, AIKEN P. Effect on depressed mood on eating among obese and nonobese dieting and nondieting persons. J Pers Soc Psychol 1981; 41: 577-85.

19. Sheppard-SaWyer C, Mcnaliy R, Fischer J. Film-induced sadness as a trigger for disinhibited eating. Int J Eat Disord 2000; 28: 215-20.

20. Heatherton TF, Herman CP, Polivy J. Effects of physical threat and ego threat on eating behavior. J Pers Soc Psychol 1991; 60: 138-43.

21. TANOFSKY-KRAFF M, WilfLEY DE, SpuRRELl E. Impact of interpersonal and ego-related stress on restrained eaters. Int J Eat Disord 2000; 27: 411-18.

22. KaPLAN HI, KaPLAN HS. A psychosomatic concept. Am J Psychother 1957; 11: 16-38.

23. MCKENNA RJ. Some effects of anxiety level and food cues on the eating behavior of obese and normal subjects: a comparison of the Schachterian and psychosomatic conceptions. J Pers Soc Psychol 1972; 22: 311-19.

24. Herman CP, Polivy J. Anxiety, restraint, and eating behavior. J Abnorm Psychol 1975; 84: 66-72.

25. Polivy J, Heatherton T, Herman P. Self-esteem, restraint, and eating behavior. J Abnorm Psychol 1988; 97: 354-6.

26. WaLis DJ, Hetherington MM. Stress and eating: the effects of ego-threat and cognitive demand on food intake in restrained and emotional eaters. Appetite 2004; 43: 39-46.

27. Heatherton TF, Striepe M, Wittenberg L. Emotional distress and disinhibited eating: The role of self. Pers Soc Psychol Bull 1998; 24: 301-13.

28. SiLva J. Sobrealimentación inducida por la ansiedad, Parte I: Evidencia conductual, afectiva, metabólica y endocrina. Ter Psicol 2007; 25: 43-55.

29. DaVidson RJ, Jackson D, KaLin N. Emotion, plasticity, context, and regulation: Perspectives from affective neuroscience. Psychol Bull 2000; 126: 890-909.

30. Silva J. Neuroanatomía funcional de las emociones. En: Labos E, Slachevsky A, Fuentes P, Manes F. Tratado de neuropsicología y neuropsiquiatría clínica. Buenos Aires: Akadia; 2008; p. 271-307.

31. Silva J, Pizzagalu D, Larson C, Jackson D, Davidson RJ. Frontal brain asymmetry in restrained eaters. J Abnorm Psychol 2002; 111: 676-81.

32. Silva J, Siachevsky A, Livacic-Rojas P. Diferencias individuales en dietantes crónicos; Influencia de los sistemas motivacionales en la alimentación. Rev Méd Chile 2006; 134: 735-42.

33. Silva J, Pizzagaui D. Cortical response to food stimulus in restrained eaters under ego-threat anxiety: an ERP source localization study. Psychophysiology 2006; 43, S92.

34. SiLva J. Sobrealimentación inducida por la ansiedad, Parte II: Un marco de referencia neurocientífico para el desarrollo de técnicas psicoterapéuticas. Ter Psicol 2008; 26: 99-115.

35. Torres W, Silva J, Silva, L. Evaluación de rasgos personales, self, esquizofrenia y estructuras de la línea media cortical. Rev Méd Chile 2007; 135: 641-8.

36. Silva J, Torres M, Ortiz M. Abnormal electrophysiological activation in schizophrenics subjects during a personal trait attribution task. Biol Res 2008; 41; 143-50.

37. Slachevsky A, Reyes P, Rojas G, Silva, J. Prefrontal cortex and control of behavior: Evidence from neuropsychological study. En: Cosmelli D, Aboitiz F, ed. From attention to goal-directed behavior. Neurodynamical, methodological and clinical trends. Berlin: Springer. En prensa.

38. Silva J, Stachevsky A. Modulación de la actividad de la corteza prefrontal como mecanismo putativo del cambio en psicoterapia. International Journal of Clinical and Health Psychology 2005; 5: 609-25.

39. Kain J, Olivares S, Castillo M, Vio F. Validación y aplicación de instrumentos para evaluar intervenciones educativas en obesidad de escolares. Rev Chil Pediatr 2001; 72, 308-18.

40. Silva J. Regulación emocional y psicopatología: El modelo de vulnerabilidad/resiliencia. Rev Chil Neuro-Psiquiat 2005; 43, 201-9. 